\title{
Toward weak confinement regime in epitaxial nanostructures: Interdependence of spatial character of quantum confinement and wave function extension in large and elongated quantum dots
}

\author{
A. Musiał, ${ }^{1,2,{ }^{*}}$ P. Gold, ${ }^{3}$ J. Andrzejewski, ${ }^{1}$ A. Löffler, ${ }^{3}$ J. Misiewicz, ${ }^{1}$ S. Höfling, ${ }^{3,4}$ A. Forchel,,${ }^{3}$ \\ M. Kamp, ${ }^{3}$ G. Sęk, ${ }^{1}$ and S. Reitzenstein ${ }^{2,3}$ \\ ${ }^{1}$ Institute of Physics, Wrocław University of Technology, Wybrzeże Wyspiańskiego 27, 50-370 Wrocław, Poland \\ ${ }^{2}$ Institute of Solid State Physics, Berlin University of Technology, Hardenbergstraße 36, D-10623 Berlin, Germany \\ ${ }^{3}$ Technische Physik and Wilhelm-Conrad-Röntgen-Research Center for Complex Material Systems, University of Würzburg, \\ Am Hubland, D-97074 Würzburg, Germany \\ ${ }^{4}$ School of Physics and Astronomy, University of St. Andrews, North Haugh, KY16 9SS St. Andrews, United Kingdom
}

(Received 15 April 2014; revised manuscript received 28 June 2014; published 31 July 2014)

\begin{abstract}
In this paper we present a comprehensive and detailed analysis of carrier/exciton wave function extension in large low-strain $\operatorname{In}_{0.3} \mathrm{Ga}_{0.7}$ As quantum dots (QDs). They exhibit rather shallow confinement potential with electron/hole localization energy below $30 \mathrm{meV}$ and confinement strength substantially weakened in comparison to typical epitaxial quasi-zero-dimensional semiconductor nanostructures. The aim of this study is to investigate the influence of different factors on the wave function (probability density distribution) for carriers or excitons in this regime, i.e., object shape anisotropy as well as strain, piezoelectricity, and Coulomb interactions, and to identify the physical mechanisms determining the properties of optical emission. To probe the wave function symmetry, polarization-resolved photoluminescence has been performed, and the spatial extensions of the corresponding probability densities have been verified in magneto-optical measurements. The observed diamagnetic coefficients in the range of (15-31) $\mu \mathrm{eV} / \mathrm{T}^{2}$ reflect large in-plane QD size. These studies also enable us to investigate the importance of light hole states admixture to the valence band ground state in such nanostructures, which can be addressed via the degree of linear polarization of emission as well as the exciton $g_{X}$ factor. The linear-polarization-resolved measurements revealed an exceptionally low exciton fine structure splitting of $5 \mu \mathrm{eV}$ on average as well as a low emission polarization degree of -0.05 , with the polarization perpendicular to the QD elongation direction dominating. The increased light hole contribution to the lowest energy hole level is reflected in the decreased exciton $g_{X}$ factor (in the range of 0-1) and is consistent with the results of the eight-band $\mathbf{k} \cdot \mathbf{p}$ modelling. Based on the temperature dependence of the diamagnetic coefficient, the problem of individual QD uniformity has additionally been discussed. To evaluate the impact of the confinment potential and the structure geometry on the optical properties of the QDs, a comparison between the investigated dots and InAs/InGaAlAs/InP quantum dashes exhibiting a much deeper confining potential is presented.
\end{abstract}

DOI: 10.1103/PhysRevB.90.045430

PACS number(s): 78.67.Hc, 73.21.La

\section{INTRODUCTION}

Due to rapidly developing nanotechnology and the related fabrication techniques, a wide variety of nano-object shapes and sizes can be easily obtained with nanoscale precision in different material systems. This gives an opportunity to engineer and control their electronic structures and the resulting optical properties. In view of quantum effects, two critically distinct domains need to be distinguished, namely the strong and weak confinement regimes $[1,2]$. The criteria for domination of the confinement effects are (i) the quantization energy being much larger than the exciton binding energy, i.e., the energy scale characteristic for Coulomb interactions between the carriers confined in a quantum dot (QD), and (ii) the volume of the exciton (defined by the exciton Bohr radius) being larger than the physical volume of the nanostructure. The strong confinement regime is typical for epitaxial QDs due to their sizes in the range of few up to tens of nanometers. The quantized electron (hole) energy level separation for typical indium arsenide QDs is in the range of 50-200 meV (25-150 meV) and $40 \mathrm{meV}$ (25 meV) for InAs/GaAs [3-7] and in the InAs/InP material

*Corresponding author: anna.musial@pwr.edu.pl system [8-10], respectively, and depends on the QD size. This should be compared to the exciton binding energy for which values in the range of $12-32 \mathrm{meV}$ and $15-33 \mathrm{meV}$, respectively, have been reported. The exciton Bohr radius for bulk InAs is $33 \mathrm{~nm}$ [11], which leads to the volume of the exciton exceeding $150000 \mathrm{~nm}^{3}$-more than 100 times larger than the actual physical volume of the typical nanostructure. Hence, the physical properties are governed by the spatial confinement, which surpasses the Coulomb effects and as a result the carrier wave function extension. In that case, the spatial symmetry of the probability density distribution follows the changes in the confining potential. For the weak confinement regime, the Coulomb interactions start to dominate over the quantum confinement effects on the single carriers. In this regime, the oscillator strength (OS) of optical transitions is governed mainly by the exciton coherence volume, which increases with the QD size as opposed to the case of strongly confined QDs [2,12-14]. This has already been proven to be advantageous for some appealing applications, e.g., those employing cavity quantum electrodynamics effects, including control of the OS allowing for tuning between strong and weak light-matter coupling $[15,16]$. For sufficiently large objects (carrier confinement being weak enough) the further increase of the nanostructure dimensions has little impact on the exciton wave function extension, and its symmetry becomes 
determined more and more by Coulomb interactions up to sizes where the three-dimensional energy quantization is preserved. Although it is relatively easy to predict the qualitative behavior of these nano-objects in the asymptotic cases (very weak and very strong confinement), it is much more difficult to make accurate predictions in the intermediate regime between weak and strong confinement without incorporating details of the actual physical system. In these cases, interpretation of experimental data is less straightforward.

The structures under study- $\mathrm{In}_{0.3} \mathrm{Ga}_{0.7} \mathrm{As}$ QDs-differ substantially from typical epitaxial QDs in terms of the confinement regime. Assuming linear interpolation between InAs $(33 \mathrm{~nm})$ and GaAs (12 nm) [11], the exciton Bohr radius for $30 \%$ indium InGaAs equals $18 \mathrm{~nm}$, which results in the exciton volume being only 2.5 times larger than the physical volume of the nanostructure. Some optical signatures confirming the weakened confining potential expected for these structures have already been reported [16,17]. Time-resolved measurements performed on both a QD ensemble, as well as on single QDs, confirmed the enhanced OS and revealed lifetimes of excitonic transitions as short as 350 ps $[16,18]$, which enabled the first observation of strong coupling between a single exciton and a single photon in a micropillar cavity [15] and makes them useful for both fundamental studies and for applications, e.g., as an efficient source of single photons. A demonstration of the weakened confinement strength was the magnetic field-driven transition from strong to weak exciton-photon coupling in this system [16]. Experiments on exciton-biexciton cascade kinetics [17], in which the biexciton lifetime was counterintuitively found to be either comparable to or longer than the exciton one, allowed another signature of weakened confining potential to be identified [12].

In this paper we focus on a detailed analysis of the interdependence of the wave function extension and the confining potential in the case of large and elongated $\operatorname{In}_{0.3} \mathrm{Ga}_{0.7} \mathrm{As} / \mathrm{GaAs}$ QDs exhibiting characteristics of confinement unique for epitaxial nanostructures. To better determine the confinement regime, eight-band $\mathbf{k} \cdot \mathbf{p}$ calculations have been performed and the characteristic energy scales of quantized single particle states and Coulomb interactions, included within the Hartree approximation, have been compared. The energy criterion confirms that the confining potential is weakened and can be classified as being in the intermediate regime with an exciton binding energy (of approximately $6 \mathrm{meV}$ ) comparable to the electron level separation $(7 \mathrm{meV})$ and much larger than the interlevel spacing of the hole states (below $0.5 \mathrm{meV})$.

The paper is organized as follows. In Sec. II, the investigated structures and the experimental setups are described. Section III is devoted to polarization-resolved measurements followed by eight-band $\mathbf{k} \cdot \mathbf{p}$ modeling aimed at revealing the symmetry of the wave function and the factors influencing it. It also contains the comparison between polarization properties of the investigated dots and InAs/InGaAlAs/InP quantum dashes (QDashes), which we use to evaluate the impact of the confinement potential depth. In Sec. IV, a detailed study of magneto-optical properties (e.g., diamagnetic coefficient and exciton $g_{X}$ factor) probing the excitonic wave function extension is presented. Section $\mathrm{V}$ concludes the paper.

\section{SAMPLE AND EXPERIMENTAL SETUP}

The investigated structures were grown by molecular beam epitaxy utilizing self-assembled formation within the StranskiKrastanow growth mode. The indium content of 30\% leads to low strain conditions due to relatively low (2\%) lattice mismatch between the QD material and the GaAs substrate. These peculiar growth conditions result in the growth of large QDs with a surface density in the range of $6-9 \times 10^{9}$ $\mathrm{cm}^{-2}$ (the latter facilitates single QD spectroscopy), which are elongated in one of the lateral directions coinciding with [1 $1 \overline{1} 0]$ the crystallographic axis. A scanning electron microscopy (SEM) image of an uncapped sample, which was grown under similar conditions as the samples studied, is presented in the bottom inset of Fig. 1. The lateral QD dimensions before overgrowth are on the order of $50-80 \mathrm{~nm}$ and $20-30 \mathrm{~nm}$, respectively, resulting in a lateral aspect ratio (LAR) of approximately two or more and height (the direction providing the strongest confinement) in the range of a few nanometers. The investigated structures were realized by submonolayer deposition of 30 alternating $\operatorname{In}_{0.12} \mathrm{Ga}_{0.88} \mathrm{As} / \mathrm{InAs}$ sequences of $0.12 \mathrm{~nm}$ and $0.03 \mathrm{~nm}$ thicknesses, respectively. The deposited material is estimated to be $4.5 \mathrm{~nm}$ thick based on the growth conditions, of which $2.7 \mathrm{~nm}$ constitutes the wetting layer (WL) [19]. Further details of the growth procedure are given elsewhere [20]. Previous optical studies on such nanostructures have revealed that not only is the spatial confinement weaker than in standard QDs due to the increased nanostructure size [17], but that also the confining potential is very shallow (in the sense of the potential depth with respect to the barrier height). This is manifested by a low-temperature emission energy difference between the QD and WL emission bands as low as $25 \mathrm{meV}$ [19]. It has important implications, especially for carrier dynamics $[18,21]$, and allows for thermally activated energy/carrier transfer between the WL and the QD ensemble as well as the WL-mediated carrier redistribution [18].

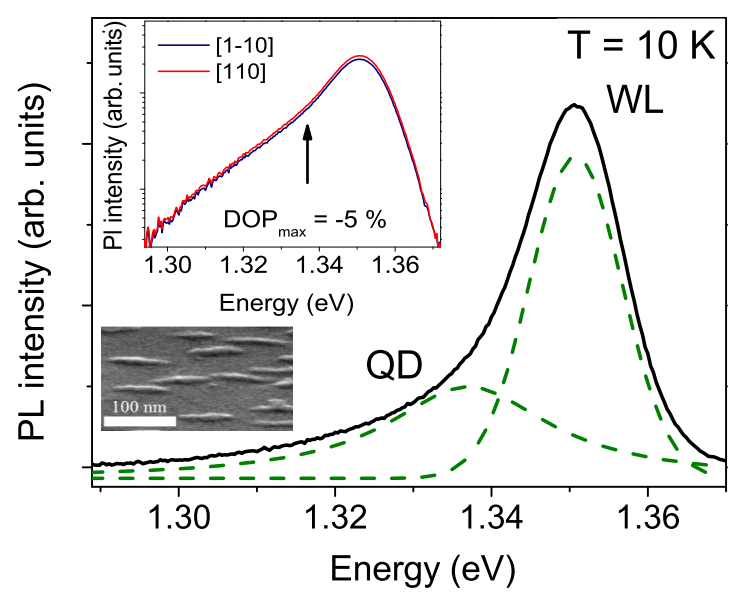

FIG. 1. (Color online) Low-temperature (10 K) ensemble photoluminescence (PL) spectra (black solid line) decomposed into two Gaussian peaks corresponding to two emission bands of the wetting layer (WL) and of $\mathrm{In}_{0.3} \mathrm{Ga}_{0.7} \mathrm{As} / \mathrm{GaAs}$ elongated QDs (green dashed lines). Top inset: polarization-resolved spectra (logarithmic scale); bottom inset: SEM image of the sample surface before overgrowth. 
Polarization-resolved photoluminescence (PL) measurements on the ensemble of nanostructures were realized on unpatterned samples using a standard far-field free-space experimental configuration with a $0.55 \mathrm{~m}$ focal length singlegrating monochromator equipped with a liquid-nitrogencooled InGaAs multichannel linear detector, and an overall spectral resolution of about $100 \mu \mathrm{eV}$. The samples were mounted in a liquid helium continuous-flow cryostat and cooled down to $5 \mathrm{~K}$. The continuous wave excitation was provided by a semiconductor laser in a nonresonant manner with a $660 \mathrm{~nm}$ wavelength beam focused on the sample surface to a spot of approximately $100 \mu \mathrm{m}$ diameter by employing long focal length optics.

To perform the measurements on a single QD, the spatial resolution was increased by using samples with submicrometer mesas patterned lithographically by electron-beam and wet chemical etching. For magneto-optical experiments and investigations on the fine structure splitting (FSS), a high resolution micro-PL setup equipped with a frequency doubled $\mathrm{Nd}$ :YAG solid state laser $(532 \mathrm{~nm})$, microscope objective (numerical aperture 0.4 ) and $0.75 \mathrm{~m}$ focal length single-grating monochromator, and a Si charge-coupled device (CCD) detector providing a spectral resolution of approximately $30 \mu \mathrm{eV}$ was used. The sample was mounted inside a magneto-optical cryostat enabling the application of constant homogeneous magnetic fields up to $5 \mathrm{~T}$ in the Faraday configuration and variation of the temperature in a wide range between $5 \mathrm{~K}$ and room temperature.

For polarization-resolved PL, rotatable quarter- or halfwave plates together with a fixed linear polarizer were used in the detection path to exclude the polarization characteristics of the spectrometer.

\section{WAVE FUNCTION SYMMETRY}

\section{A. Degree of linear polarization of the surface emission}

An important figure of merit in the following experiments is the degree of linear polarization (DOP) of surface emission obtained at the maximum of the QD ensemble emission band. It describes the dominance of one of the linear polarization components over another:

$$
\mathrm{DOP}=\frac{I_{I I}-I_{\perp}}{I_{I I}+I_{\perp}},
$$

with $I_{I I}$ and $I_{\perp}$ being the intensity of surface emission polarized in the direction longitudinal and transverse to the structure elongation. Lowering the in-plane confinement potential symmetry results in a ground hole state of a mixed character (of light and heavy hole-lh and hh, respectively), which leads to a lifting of the ground state degeneracy and two unequal linearly polarized components [22]. The emission intensity ratio of the two states is determined by the degree of the valence band mixing. The cause of the valence band mixing can be complex, i.e., anisotropic strain distribution [22,23], nanostructure shape anisotropy (proven for strain-free QDs grown by droplet epitaxy method [24]), and increased nanostructure height resulting in decreased lh-hh energy separation [25,26]. In the first approximation two linearly polarized components are orthogonal and in the direction determined by the structure geometry. When more subtle effects on the single dot level are taken into account (i.e., local strain distribution in the vicinity of an individual QD), both the direction and the angle between different components can vary significantly from dot to dot [27-29]. Measurements presented in this subsection have been performed on the QD ensemble to average these effects. The preferential directions of linear components have been carefully checked and appear to coincide with the crystallographic axes parallel and perpendicular to the structure elongation direction [1 10$]$.

In Fig. 1, a low-temperature $(10 \mathrm{~K}) \mathrm{PL}$ spectrum is presented. Two emission bands can be resolved: the PL band related to the QD ensemble with an inhomogeneous broadening of approximately $30 \mathrm{meV}$, reflecting the size and composition distribution, and the WL-related PL. The emission bands overlap significantly due to shallow confining potential of the QDs and the low-energy tail of the WL density of states related to the thickness/composition fluctuations of the WL quantum well. Polarization-resolved measurements were performed at $5 \mathrm{~K}$ and reveal DOP values at the maximum of the QD ensemble emission on the order of $-0.05 \pm 0.01$ without any significant dependence on the emission energy. In the top inset of Fig. 1, PL spectra for linear polarization axes along [110] and [110] direction are presented in logarithmic scale. Taking the pronounced shape asymmetry of the $\mathrm{In}_{0.3} \mathrm{Ga}_{0.7} \mathrm{As}$ QDs into account (bottom inset of Fig. 1), DOP values are surprisingly low. The negative sign of DOP [according to the definition in Eq. (1)] is also rather counterintuitive as it indicates that the intensity of the polarization component perpendicular to the QD elongation is higher than that for the light polarized parallel to it. Further, we have found a very weak dependence of DOP on both the excitation power and temperature (not shown here). A slight decrease of the absolute value of DOP (down to 0.03 at $50 \mathrm{~K}$ ) for higher temperatures can be understood when the sequential contribution of higher energy states is taken into account [29]. Indeed, this trend was verified in the calculations described below, which show that for the typical elongated QD geometry, DOP for higher energy transitions with significant contribution to the emission (sufficiently large $\mathrm{OSs}$ ) varies in the range of -0.06 to -0.002 . One possible simple explanation for the low DOP value could be that the QDs have almost symmetrical shape. This is ruled out here as being contradictory to the reported geometrical properties of low In content InGaAs QDs on GaAs substrate in that range of compositions [20,30,31]. Another possibility is to relate the observed DOP value to localization effects preventing the wave function from extending over the entire QD volume, thereby increasing its symmetry. Potential fluctuations on a spatial scale smaller than the nanostructure size leading to carrier trapping have been previously observed in 1D systems [32,33] and strongly elongated quasi-zero-dimensional structures in the InAs/InGaAlAs/InP material system [34]. However, our previous experimental results on $\mathrm{In}_{0.3} \mathrm{Ga}_{0.7} \mathrm{As} / \mathrm{GaAs} \mathrm{QDs}$ do not support this scenario. The analysis of the PL thermal quenching on the single QD level shows that only in rare cases an activation energy on the order of a few milli-electronvolts appears, which could be interpreted as a fingerprint of additional localization within an individual nanostructure [35]. Finally, the dependence of DOP on temperature is characteristic for uniform nanostructures [34]. All these arguments, 
combined with the temperature dependence of the diamagnetic coefficient discussed in Sec. III C, lead to the conclusion that in this case additional localization within the nanostructure is rather unlikely and should not dominate the effective optical response of the QD ensemble and, hence, cannot explain the low experimental DOP values.

To understand the physical effects behind the observed low and negative value of DOP, numerical simulations have been performed. To model the polarization properties of QDs, an accurate description of the band mixing is required; therefore, the full eight-band $\mathbf{k} \cdot \mathbf{p}$ Hamiltonian was used, including realistic strain distribution and piezoelectric effects (up to the second order). A more detailed analysis of the utilized theoretical approach can be found elsewhere [36,37]. To study the influence of the excitonic effects, the Coulomb interactions between carriers confined in the QD have been included within the Hartree model. This leads to the modification in the wave function and, as a result, the OS of respective transitions and correction in the emission energy by the exciton binding energy. To obtain the dependence of DOP on the LAR, the OS of the optical transitions for different polarization components has been calculated. The QD length $L$ was varied, while the base width $W$ and height $H$ were left unchanged and equal to $30 \mathrm{~nm}$ and $4.3 \mathrm{~nm}$, respectively, in agreement with the existing structural data [20]. A lens-shaped QD geometry and a 2.7-nm-thick WL have been assumed based on available data for the InGaAs/GaAs material system [38]. The scheme of the system geometry (QD: red; WL: blue) used in the calculations together with numerical box (gray) is depicted in the inset of Fig. 2.

The results of the calculated DOP dependence on the QD lateral geometry including different effects are shown in Fig. 2. In the first attempt, the excitonic effects have not been included in these considerations. This is justified because the valence

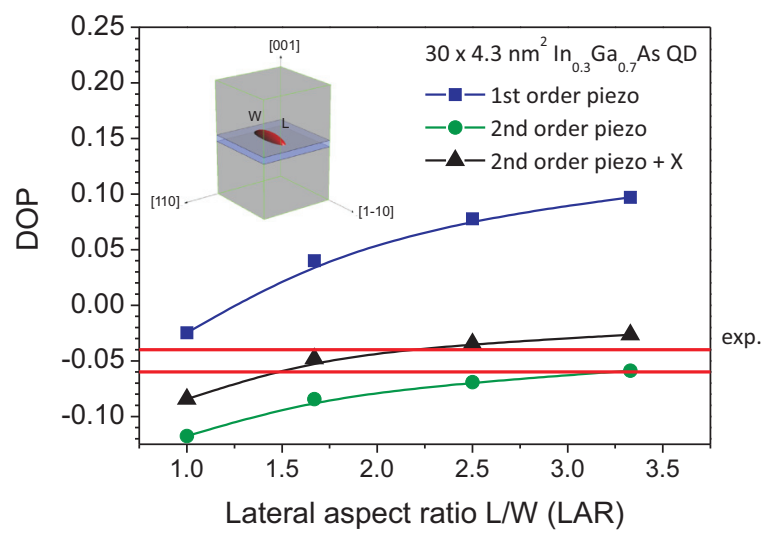

FIG. 2. (Color online) Full eight-band k·p calculation results of the DOP dependence on LAR for $30 \times 4.3 \mathrm{~nm}^{2} \mathrm{In}_{0.3} \mathrm{Ga}_{0.7}$ As QDs including only first-order piezoelectric effect (blue squares), both first- and second-order piezoelectric effect (green dots) in the single particle picture, Coulomb interactions (black triangles) with solid lines being only guides to the eye, and the range of experimentally observed DOP values marked by red horizontal lines. Inset: Schematic representation of an $\mathrm{In}_{0.3} \mathrm{Ga}_{0.7}$ As QD structure geometry used in eight-band $\mathbf{k} \cdot \mathbf{p}$ calculations showing quantum box size (gray), WL (blue), and QD (red). band mixing primarily determines the polarization properties of emission. The admixture of the lh states into the hole ground state is in the range of $1.9-2.7 \%$ (depending on the LAR); as a result, DOP is nonzero. If the first-order piezoelectric effect exclusively is included, a negative value of DOP can be reproduced only for isotropic QDs, as can be seen in Fig. 2 (blue squares), for which DOP equals to -0.025 (absolute value smaller than observed experimentally). With increasing length of the QD, the DOP changes its sign and increases smoothly up to 0.1 for QD lengths as large as $100 \mathrm{~nm}$ (LAR above 3), i.e., certainly above the typical elongation of the investigated structures. In the case of preserved QD symmetry, the piezoelectric field favors the [110] direction leading to negative DOP values, but as the QD elongation is increased, the influence of the confining potential anisotropy starts to dominate. Clearly the linear piezoelectric effect cannot explain the outcome of polarization-resolved measurements. Including second-order piezoelectric effects appears to be sufficient to explain the negative DOP in a wider range of geometries (green dots in Fig. 2) [39-41]. Even though there is no quantitative correspondence to the experimental data in the realistic LAR range, we could conclude that the nonlinear piezoelectric field can be identified as the physical mechanism enforcing the negative sign of DOP. As the QD gets elongated, the absolute value of DOP decreases (the emission becomes less polarized). This is due to the increasing extension of the wave function in the elongation direction. Thus, the observed DOP is a result of the interplay between these two major effects, where the elongation cannot compensate for the inherent piezoelectric field anisotropy.

Adding Coulomb interactions to this picture does not change the results qualitatively. It shifts the whole DOP dependence upwards by around 0.04 as it is an additional factor compensating for the piezoeletricity-induced wave function anisotropy (black triangles in Fig. 2). This is explained by Coulombic attraction between electrons and holes and the overall tendency for the probability densities to symmetrize. This correction appears to be sufficient to account for the experimental observations and enables us to deduce a LAR in the range of 1.5-2, in agreement with expected in-plane QD geometry.

To have a better insight into DOP behavior and because the phenomena can be qualitatively understood essentially in the single particle picture, electron and hole probability distributions for symmetric (top panel) and anisotropic QD shape (bottom panel) are presented in Fig. 3(a). Here the black solid lines indicate the contour of the QD base shape. The calculations reveal that the hole wave function is very anisotropic and laterally extends along the [110] direction. Moreover, in both cases its probability distribution shows maxima on the edges of the QD (due to piezoelectric part of the potential), which is responsible for the nonzero and negative values of DOP, even for symmetric QDs. An elongation of the QD increases the wave function extension in the elongation direction (i.e., [1 $1 \overline{1} 0]$ direction), which makes it more symmetric and effectively lowers the polarization degree. On the contrary, the electron probability density is confined to the center of the QD, symmetric for isotropic in-plane geometry, and follows the changes in the confinement potential in the case of laterally extended QDs. This comparison highlights 
(a)

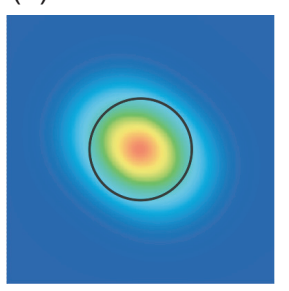

electron

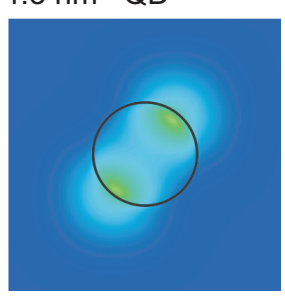

hole

$30 \times 75 \times 4.3 \mathrm{~nm}^{3} \mathrm{QD}$

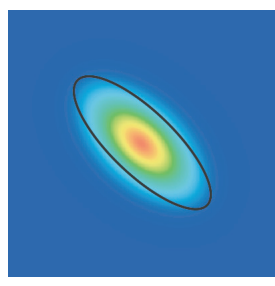

electron

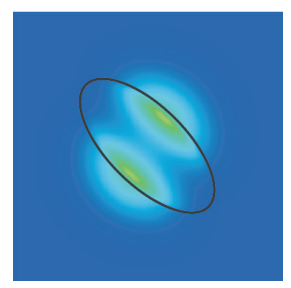

hole

(b) $30 \times 50 \times 4.3 \mathrm{~nm}^{3}$

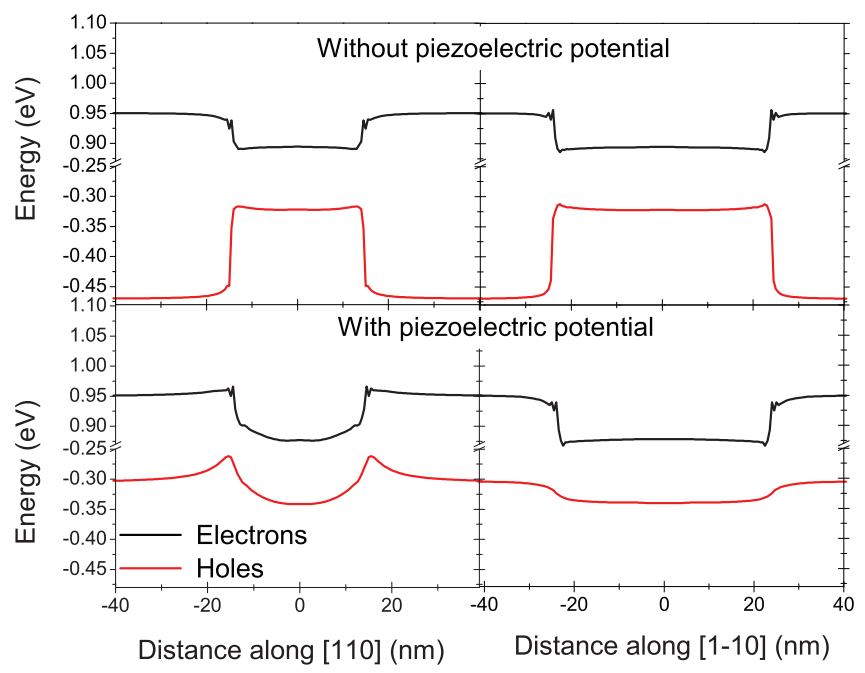

FIG. 3. (Color online) (a) Calculated electron (left) and hole (right) density function probabilities in the vertical (growth) direction for $\mathrm{In}_{0.3} \mathrm{Ga}_{0.7} \mathrm{As} / \mathrm{GaAs}$ QDs on the WL for a symmetric (top) and asymmetric (bottom) QD in the single particle picture. The black contour shows QD shape/size assumed in the calculations. (b) Calculated confining potential cross sections along the [110] (left) and [1 10$]$ directions without (top panel) and with (bottom panel) piezoelectric potential up to the second order for $30 \times 50 \times 4.3 \mathrm{~nm}^{3}$ QD geometry.

the impact of the confining potential details on the symmetry of the electron and hole wave functions. Due to the combined effect of the barrier potential height (band gap discontinuity), strain-induced band offset and piezoelectric potential, rather shallow confinement is obtained. The most important difference regarding the piezoelectric potential is that its inclusion makes the confinement potential deeper for electrons and shallower for the holes. This can be clearly seen in Fig. 3(b) where the cross sections of confining potential along the short (left) and long (right) QD axes without (top panel) and with (bottom panel) the piezoelectric potential are compared. As a consequence, the symmetry of the electron wave function is determined by the QD physical shape. The situation differs for the hole states with anisotropic effective mass, which are much more susceptible to the piezoelectric field, which can overcome the spatial confinement and defines the main optical axis.

To emphasize the influence of confinement potential depth on the polarization properties of emission, we have compared the investigated structures with other elongated nanostructures-InAs/InGaAlAs/InP QDashes (LAR exceeding 5) [42-45]. Such InP-based structures exhibit much deeper confinement potential for both types of carriers, i.e., by definition expected to be much less sensitive to the peculiarities of the confining potential shape. But on the other hand, the QDashes are similar to the investigated $\operatorname{In}_{0.3} \mathrm{Ga}_{0.7} \mathrm{As} / \mathrm{GaAs}$ QDs with respect to the lack of in-plane rotational symmetry and the resulting $\mathrm{lh}$ contribution to the excitonic states. Much higher DOP — in the range of 0.15 up to 0.3 - was observed experimentally $[34,46]$. This can be traced back to a larger $\mathrm{lh}$ contribution to the valence band ground state, in the range of 3.2-5.1\% for QDash structures. In the case of the investigated $\mathrm{In}_{0.3} \mathrm{Ga}_{0.7}$ As QDs, it equals only $1.9-2.7 \%$, depending on the LAR. Numerical calculations similar to those presented in this paper predict DOP reaching up to 0.3 for strongly anisotropic objects, corresponding well with the experimental data [37]. Negative DOP values have not been observed experimentally or predicted theoretically. This striking difference can be attributed to the wave function symmetry, which in this case corresponds better to the shape of the nanostructure due to stronger confinement of the carriers inside a relatively deep potential well. The activation energy with respect to the WL for a typical QDash is about $118 \mathrm{meV}$ (54 meV) for electrons (holes) [47], in comparison to $29 \mathrm{meV}$ (19.6 meV) calculated for the investigated $\mathrm{In}_{0.3} \mathrm{Ga}_{0.7}$ As QDs. The presented considerations prove that in the case of shallow confining potential, the wave function sensitivity to the details of QD shape is reduced and can be further diminished by the weakened confinement. This brings one to the conclusion that for quasi-zero-dimensional systems where the carriers are not strongly confined, the interpretation of polarization-resolved measurements as well as predictions on the polarization properties of emission are not straightforward. This is very important in view of applications, especially in optoelectronics or nanophotonics, because of the demand for some devices to be entirely polarization insensitive (e.g., optical amplifiers) and others having preferable polarization direction (e.g., lasers or single photon sources for applications in the field of quantum cryptography). There are solutions based on additional external controlling systems changing the polarization state or filtering out the unwanted components [48], but they are always accompanied by increased losses (which require being balanced by increased source efficiency-commonly already pushed to the limits), more complicated fabrication processes, or significantly less compact device designs. It would be preferable if the polarization of light suitable for specific applications could be selected based on the design of the active region alone.

\section{B. Exciton FSS}

In the ideal case of full in-plane symmetry and if the electron-hole exchange interaction is neglected, the hh exciton 
(a)

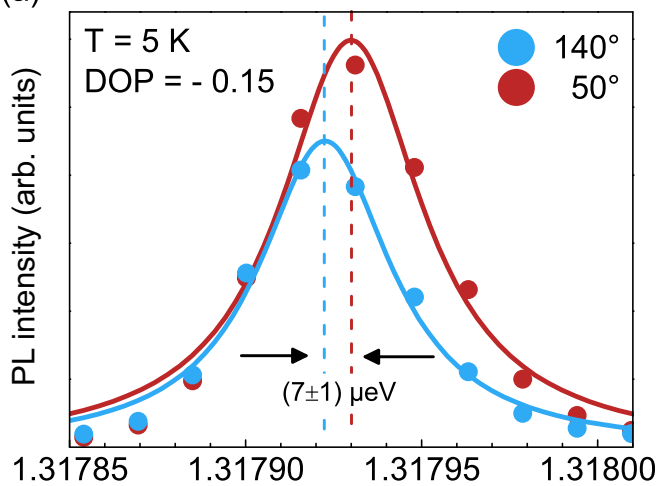

(c)

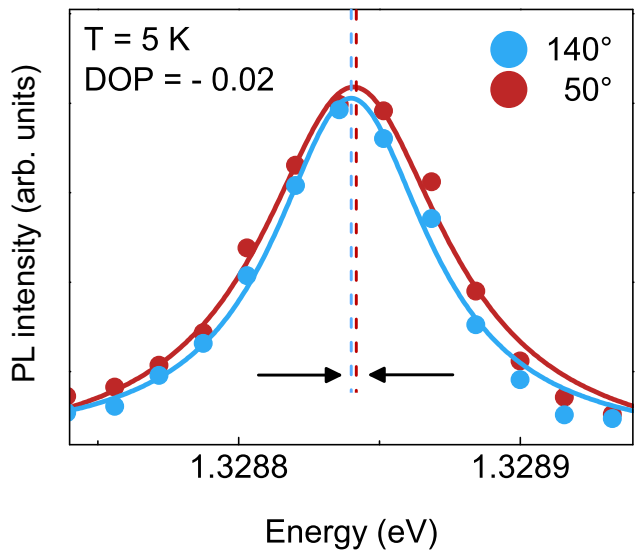

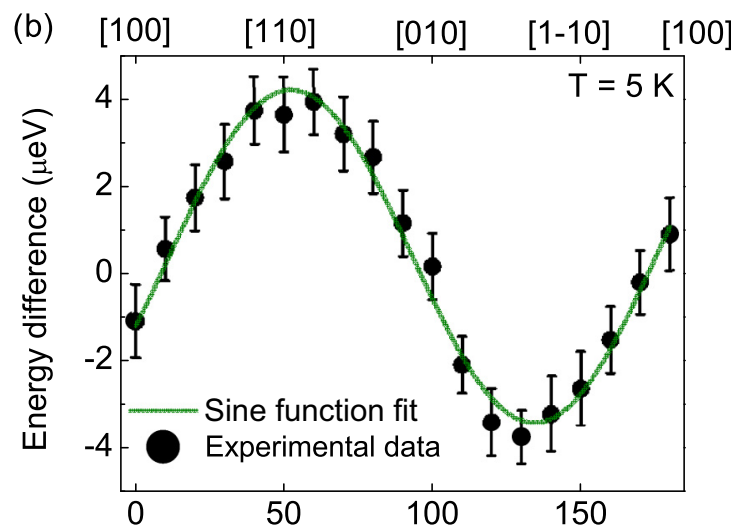

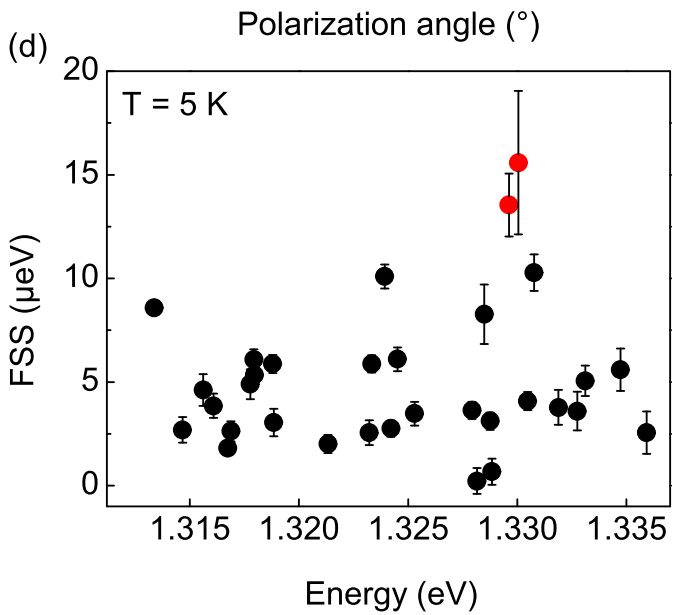

FIG. 4. (Color online) $\mu \mathrm{PL}$ spectra of two single QDs with DOPs of (a) -0.15 and (c) -0.02 , respectively, measured for two orthogonal polarization directions. (b) Corresponding polarization angle emission energy dependence of (a), from which the FSS has been extracted. (d) FSS dependence on exciton emission energy obtained experimentally with error bars estimated based on single emission line fitting procedure uncertainty.

ground state is fourfold degenerate due to two possible $z$ components of the electron spins $( \pm 1 / 2)$ and the hole angular momenta $( \pm 3 / 2)$. Two of these exciton states carry angular momentum of two and are dipole forbidden (dark excitons), whereas the remaining two are circularly polarized (angular momentum equals \pm 1 ) and optically active (bright excitons). The electron-hole exchange interaction lifts this degeneracy completely and mixes states with well-defined angular momenta, which results in linearly polarized exciton eigenstates [49]. The energy splitting between the two bright excitons, so-called FSS resulting from the anisotropic part of this interaction [50], is caused by lowering the symmetry of the confining potential. When considered on the atomistic level, it can be related to the crystal lattice symmetry and its disturbance due to atomic strain relaxation at the interfaces [7,51]. The resulting splitting is being referred to as intrinsic FSS and is generally on the order of a single micro-electron-volt. Other mechanisms leading to lowering of the confining potential symmetry are shape asymmetry and strain-induced piezoelectric fields [5]. The FSS can be determined experimentally in polarization-resolved PL because the nondegenerate optically active excitonic states have orthogonal linear polarizations. This can be seen in
Figs. 4(a) and 4(c) where $\mu \mathrm{PL}$ spectra measured for two orthogonal polarization directions are presented. In both cases, the lower energy component has lower intensity and corresponds to the polarization axis approximately parallel to the QD elongation direction ( $5^{\circ}$ deviation), which is in good agreement with polarization-resolved measurements on the QD ensemble. The determined DOP of -0.15 and -0.02 , for examples from Figs. 4(a) and 4(c), respectively, underlines the importance of the strain distribution in the vicinity of the QD and the differences between the dots within the ensemble (size and LAR), which result in a DOP distribution. Because of extremely low FSS values, $7 \mu \mathrm{eV}$ and $1 \mu \mathrm{eV}$, for cases from Figs. 4(a) and 4(c), respectively, a series of emission spectra for different polarization angles was measured, and the energy dependence on the polarization angle was examined, as shown in Fig. 4(b), for the QD from Fig. 4(a). The value of FSS for the QD shown in Fig. 4(c) is within the experimental error $(1 \mu \mathrm{eV})$; therefore, the polarization dependence of emission is not shown here. All these experiments were performed at $10 \mathrm{~K}$ and low excitation power density to ensure mainly the single exciton emission and to reduce the probability of exciting other complexes or higher states. A statistical analysis performed on 32 single exciton lines reveals an average FSS of $5 \mu \mathrm{eV}$, as can 
be seen in Fig. 4(d), where the FSS is plotted as a function of the QD emission energy. The result is again counterintuitive when taking only the pronounced in-plane asymmetry of the $\mathrm{In}_{0.3} \mathrm{Ga}_{0.7} \mathrm{As}$ QDs into account. We would like to point out that the average FSS is significantly lower than values observed for standard InGaAs QDs in a GaAs matrix [49,5254]. It is even close to the values observed for strainfree GaAs/AlGaAs QDs [55] or $\operatorname{In}(\mathrm{Ga}) \mathrm{As}$ QDs grown on (111)-oriented GaAs [56-59], which makes the $\operatorname{In}_{0.3} \mathrm{Ga}_{0.7} \mathrm{As}$ appealing for the generation of polarization entangled photon pairs using the biexciton-exciton cascade $[59,60]$. The reason for the confinement potential asymmetry not to be manifested in increased FSS may be the same as for the low DOP of emission-the shallow confining potential causing lower sensitivity of the wave function symmetry to the details of the QD shape due to its extension being much smaller than the potential size. This result is significant, not only in view of fundamental research, pointing out that very small FSS does not necessarily require highly symmetric QDs, but also in consideration of the applications, especially those relying on photon indistinguishability and entanglement [61-64].

Additionally, no correlation between the magnitude of the FSS and the exciton emission energy has been observed, as presented in Fig. 4(d) [65]. Normally the spectral distribution of the FSS is used to examine its dependence on the QD size [66-68], but in the case of low In content InGaAs/GaAs QDs, it is not straightforward as the emission energy is driven by more than one factor and the influence of each of them is not easy to extract due to the self-organized character of QD formation resulting in per-dot differences in shape, size, and composition. The study of InGaAs QD samples differing strongly in composition has revealed a clear correlation between the QD size and the In content - the higher the In content, the smaller the size of the nanostructure due to increased lattice mismatch and the resulting strain. This trend can also be observed on a smaller scale, i.e., within one QD sample with a given nominal In content. We discuss this in more detail in Sec. IV A. Even with this additional information, predictions of the FSS dependence on the exciton emission energy cannot be easily made due to the complex influence of In on the emission energy. The In content changes the bandgap energy as well as the lattice constant of the InGaAs alloy. The former influences the emission energy directly, and the latter affects the lattice mismatch with the GaAs substrate and varies the strain, which further determines the QD size and, as a result, the part of the emission energy related to the electronic structure of the QD. These effects are competing as they induce opposing changes in the emission energy. The secondary effect is the tendency of In to segregate, which is also composition dependent.

\section{WAVE FUNCTION EXTENSION}

\section{A. Diamagnetic coefficient}

To examine in detail the wave function extension, we performed single QD magneto-optical studies up to $5 \mathrm{~T}$. From the energy position of individual QD emission lines we determined the diamagnetic coefficient assuming a quadratic energy shift as a function of magnetic field. This is justified in our experimental conditions (weak magnetic fields) due to much smaller cyclotron energy when compared to the exciton binding energy of the investigated structures $[4,9,69,70]$. In this case, the diamagnetic coefficient $\kappa$ can be expressed as follows [71]:

$$
\kappa=\frac{e^{2}}{8}\left(\frac{\left\langle\rho_{e}^{2}\right\rangle}{m_{e}}+\frac{\left\langle\rho_{h}^{2}\right\rangle}{m_{h}}\right),
$$

where $\left\langle\rho^{2}\right\rangle$ is the average quadratic extension of the wave function, $m_{e}$ stands for the in-plane effective mass of an electron, and $m_{h}$ for the average hole effective mass. The exciton wave function has been probed experimentally, and by substituting the carrier effective mass with the exciton reduced mass the mean lateral extension of the exciton wave function $2 \sqrt{\left\langle\rho_{X}^{2}\right\rangle}$ has been obtained. We would like to point out that the diamagnetic coefficient probes only the wave function average extension (radius) in the smallest direction perpendicular to the orientation of the applied magnetic field but does not give any information about its symmetry, which should be deduced independently.

Diamagnetic coefficients determined experimentally for $\mathrm{In}_{0.3} \mathrm{Ga}_{0.7} \mathrm{As}$ elongated QDs in the Faraday configuration are in the range of $15-31 \mu \mathrm{eV} / \mathrm{T}^{2}$ [Fig. 5(c)], which are significantly larger than values reported for other $\operatorname{In}(\mathrm{Ga}) \mathrm{As}$ QDs [16,72-74]-typically less than several micro-electronvolts per square of the magnetic field. This result is not surprising taking into account the low In content leading to an increased exciton Bohr radius in these nanostructures [15-17]. A more detailed investigation has revealed an increasing diamagnetic coefficient as a function of the emission energy, as presented in Fig. 5(c). The observed tendency cannot be simply related to the size of the QDs because in that case the opposite trend would be expected-smaller QDs with higher emission energy should exhibit lower diamagnetic coefficients due to stronger wave function confinement. Thus, changes in emission energy seem to be of more complicated origin being a result of the interplay between the QD size and the indium content. The increase of the diamagnetic coefficient for larger emission energy suggests that the emission in this spectral range originates preferentially from larger QDs within the ensemble of emitters. This can be explained by a lower In content of these QDs, which is in agreement with the trend observed for higher In content variation: indium-rich QDs with 60\% In are smaller than 30\% In nanostructures mainly due to lowering the lattice mismatch with respect to the GaAs matrix [20], but they emit at longer wavelengths as a result of band gap shrinkage due to the larger In mole fraction. As a result, the emission energy is higher for larger QDs, which is in qualitative agreement with the trend observed in magneto-optical experiments. The dependence of the diamagnetic coefficient as a function of the emission energy can be interpreted as simultaneous size and In content variation within the QD ensemble.

Furthermore, the diamagnetic shift as a function of magnetic field for various temperatures in the range of 7 to $35 \mathrm{~K}$ has been measured. The respective dependences of the diamagnetic shifts as a function of the square of the magnetic field for selected temperatures $(7 \mathrm{~K}, 20 \mathrm{~K}$, and $35 \mathrm{~K}$ ) are presented in Fig. 5(a) together with a linear fit to the 
(a)

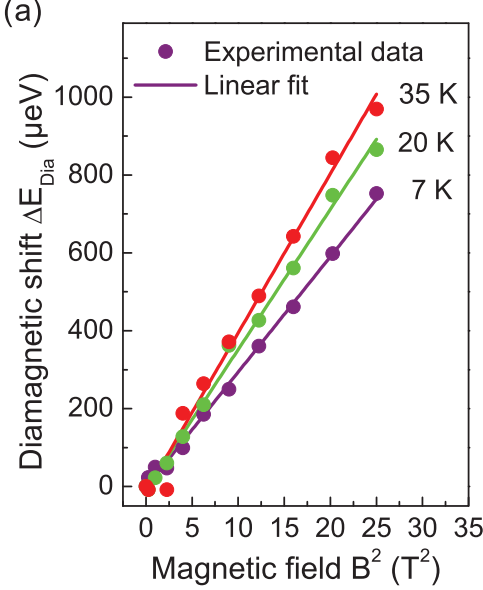

(b)

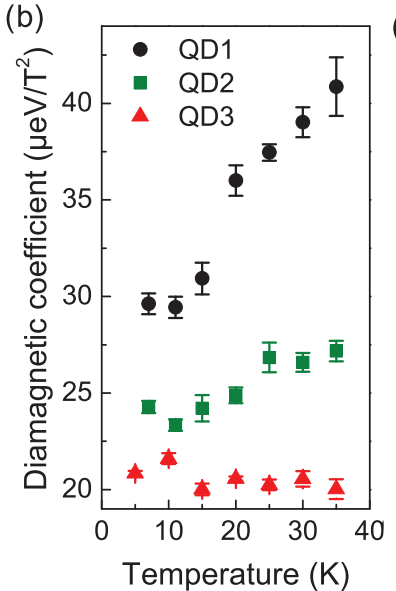

(c)

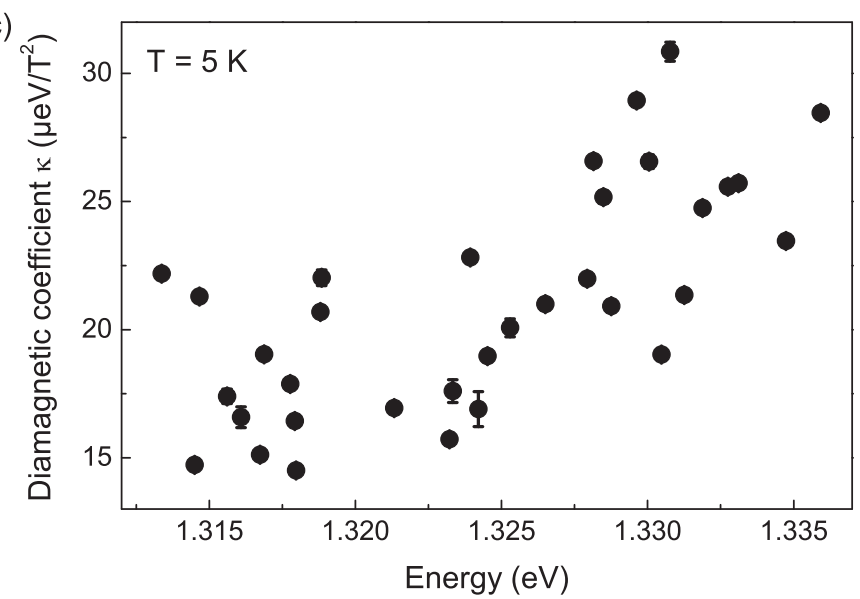

FIG. 5. (Color online) (a) Magnetic field dependence of diamagnetic shift for a single elongated $\operatorname{In}_{0.3} \mathrm{Ga}_{0.7} \mathrm{As} / \mathrm{GaAs} \mathrm{QD}$ for different temperatures in the range from 7 to $35 \mathrm{~K}$ with linear fit (straight line) to experimental data (dots). (b) Temperature dependence of the diamagnetic coefficient for three exemplary QDs exhibiting qualitatively different behavior. (c) Experimentally obtained dependence of diamagnetic coefficient on emission energy.

experimental data. Further study revealed distinctive behavior of the diamagnetic coefficient as a function of temperature for different QDs. In Fig. 5(b), examples of three qualitatively different behaviors are shown, i.e., the temperature dependence of the diamagnetic coefficient is plotted for three QDs. These different types of behavior can be classified based on the low-temperature value of the diamagnetic coefficient. For QDs showing the highest diamagnetic coefficients, a sharp increase with temperature has been observed, e.g., from 30 up to $41 \mu \mathrm{eV} / \mathrm{T}^{2}$ at $35 \mathrm{~K}$ [QD1 in Fig. 5(b)]. For the intermediate case of $24 \mu \mathrm{eV} / \mathrm{T}^{2}$, the increase is much more subtle, about $10 \%$ in the same temperature range (QD2). In the case of the smallest diamagnetic coefficient $-21 \mu \mathrm{eV} / \mathrm{T}^{2}-$ no clear temperature dependence can be observed (QD3). All these observations can be understood as follows: a large value of the diamagnetic coefficient corresponds to a large wave function extension resulting from weaker confinement for carriers than in the case of smaller QDs. An abrupt change in the diamagnetic coefficient with temperature is a manifestation of spreading the probability density over the entire QD structure from being confined at a local minima of potential originating from, e.g., QD height or In content fluctuations on the scale of individual QDs. The latter is in agreement with premises from PL-quenching measurements on individual QDs where small activation energy has been observed in some cases [35]. On the other hand, when the low temperature value of the diamagnetic coefficient is relatively small and it does not change with temperature, we are dealing with a QD exhibiting stronger quantum confinement (smaller size lowering the probability of additional trapping centers appearing), and the wave function is extended over the entire QD volume already at $5 \mathrm{~K}$, which is why the temperature has no significant impact on the diamagnetic coefficient.

\section{B. Excitonic $g_{X}$ factor}

Magneto-optical measurements on single QDs have also enabled us to extract the excitonic Lande $g_{X}$ factor from the Zeeman splitting dependence on the magnetic field. Excitonic
$g_{X}$ factors obtained for $\operatorname{In}_{0.3} \mathrm{Ga}_{0.7}$ As QDs under the assumption of a linear dependence of the Zeeman splitting on the magnetic field are in the range of 0-1 (Fig. 6), significantly lower than for In-rich smaller QDs for which the values of approximately 3 have been reported [72]. Also, the interdependence between the diamagnetic coefficient and $g_{X}$ factor values character is reversed-larger $g_{X}$ factors are characteristic for structures exhibiting low diamagnetic coefficients (Fig. 6). This can be explained by an increased lh component in the valence band states of elongated QDs (weaker confinement of holes enhances further the states mixing in the valence band). The excitonic Landé $g_{X}$ factor is composed of hole and electron contributions. It is well established that the hole $g_{h}$ factor is much more sensitive to the details of QD size, shape, and composition than the electron $g_{e}$ factor [75-77]. For the in-plane symmetric flat nanostructures, the hh component with higher $g_{\text {hh }}$ factor dominates (especially in self-assembled structures under the compressive strain), but the admixture of the lh states increases with increasing QD elongation resulting in lowering the hole $g_{h}$ factor and, as a consequence, also the excitonic $g_{X}$ factor.

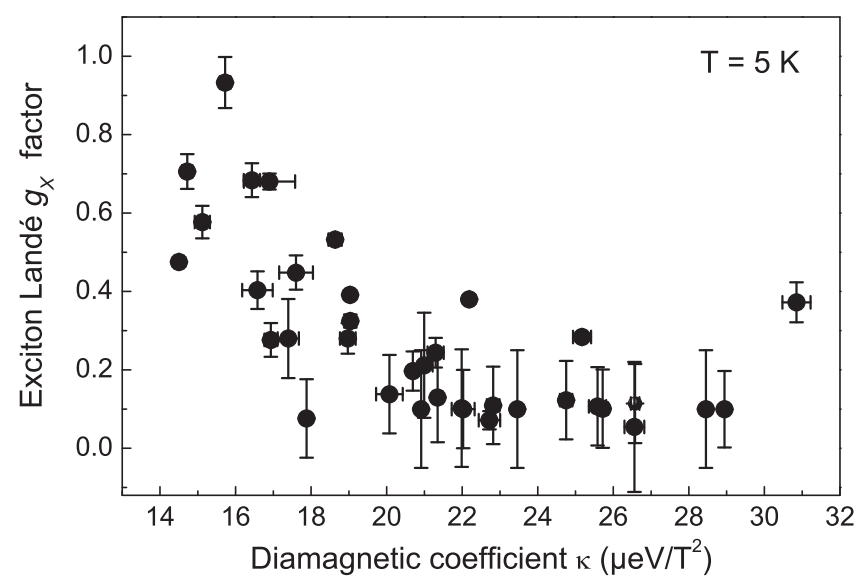

FIG. 6. Experimental dependence of the exciton Landé $g_{X}$ factor on the diamagnetic coefficient for elongated $\operatorname{In}_{0.3} \mathrm{Ga}_{0.7}$ As QDs. 


\section{CONCLUSIONS}

High resolution optical and magneto-optical spectroscopy combined with numerical calculations based on eight-band $\mathbf{k} \cdot \mathbf{p}$ theory has been used to examine the wave function extension and its symmetry in large $\operatorname{In}_{0.3} \mathrm{Ga}_{0.7} \mathrm{As} / \mathrm{GaAs} \mathrm{QDs}$. In spite of the significant shape anisotropy of these nanostructures, we detected the smallest values of the ground state exciton FSS when compared to any epitaxial InGaAs/GaAs QDs on (001)-oriented GaAs substrate, which is highly attractive for the generation of polarization entangled photon pairs. Despite pronounced shape asymmetry, the DOP of the $\operatorname{In}_{0.3} \mathrm{Ga}_{0.7} \mathrm{As}$ QDs has a surprisingly low value, in the range of a single percentage, with the main optical axis perpendicular to the QD elongation. This has been attributed to the anisotropy of the nonlinear piezoelectric field, which dominates over the effect of spatial confinement symmetry. In addition, shallow confining potential makes the wave function less sensitive to the QD shape changes, especially for the hole states. Polarization-resolved measurements show that the high in-plane anisotropy is not sufficient for DOP to be large. In the case of shallow and weakened confining potential, DOP cannot be used as a probe of lateral shape or confining potential symmetry as it rather reflects the symmetry of the wave function, which is driven by more factors than the structure shape itself. In comparison to more common higher In content InGaAs QDs, the obtained diamagnetic coefficient is larger, and the $g_{X}$ factor is smaller. The former confirms larger wave function extension, whereas the latter is related to increased valence band mixing (decreased $g_{h}$ factor) resulting from the QD shape anisotropy. The obtained exciton wave function extensions prove a weakened confining potential implying non-negligible influence of the Coulomb interactions in such dots.

\section{ACKNOWLEDGMENTS}

The work has financially been supported by the Grant No. 2011/02/A/ST3/00152 of the National Science Center of Poland. The experiments have partially been performed within the laboratory infrastructure at Wroclaw University of Technology financed by the Polish Ministry of Science and Higher Education Grant No. 6167/IA/119/2012. A.F. and J.M. gratefully acknowledge the Foundation for Polish Science (FNP) and Deutsche Forschungsgemeinschaft (DFG) for financial support within the COPERNICUS Award.
[1] Semiconductor Quantum Dots, edited by L. Banyai and S. W. Koch (World Scientific Publishing, New York, 1993).

[2] R. C. Iotti and L. C. Andreani, Phys. Rev. B 56, 3922 (1997).

[3] C. Pryor, Phys. Rev. B 57, 7190 (1998).

[4] Ph. Lelong, O. Heller, and G. Bastard, Solid State Electron. 42, 1251 (1998).

[5] M. Grundmann, O. Stier, and D. Bimberg, Phys. Rev. B 52, 11969 (1995).

[6] M. Grundmann, N. N. Ledentsov, O. Stier, D. Bimberg, V. M. Ustinov, P. S. Kopév, and Zh. I. Alferov, Appl. Phys. Lett. 68, 979 (1996).

[7] G. Bester and A. Zunger, Phys. Rev. B 71, 045318 (2005).

[8] R. J. Warburton, B. T. Miller, C. S. Dürr, C. Bödefeld, K. Karrai, J. P. Kotthaus, G. Medeiros-Ribeiro, P. M. Petroff, and S. Huant, Phys. Rev. B 58, 16221 (1998).

[9] G. A. Narvaez, G. Bester, and A. Zunger, Phys. Rev. B 72, 245318 (2005).

[10] M. Syperek, Ł. Dusanowski, J. Andrzejewski, W. RudnoRudziński, G. Sęk, J. Misiewicz, and F. Lelarge, Appl. Phys. Lett. 103, 083104 (2013).

[11] Handbook Series on Semiconductor Parameters, edited by M. Levinshtein, S. Rumyantsev, and M. Shur (World Scientific, New York, 1996).

[12] M. Wimmer, S. V. Nair, and J. Shumway, Phys. Rev. B 73, 165305 (2006).

[13] T. Takagahara, Phys. Rev. B 36, 9293 (1987).

[14] E. Hanamura, Phys. Rev. B 37, 1273 (1988).

[15] J. P. Reithmaier, G. Sek, A. Löffler, C. Hofmann, S. Kuhn, S. Reitzenstein, L. V. Keldysh, V. D. Kulakovskii, T. L. Reinecke, and A. Forchel, Nature 432, 197 (2004).

[16] S. Reitzenstein, S. Münch, P. Franeck, A. Rahimi-Iman, A. Löffler, S. Höfling, L. Worschech, and A. Forchel, Phys. Rev. Lett. 103, 127401 (2009).
[17] G. Sęk, A. Musiał, P. Podemski, M. Syperek, J. Misiewicz, A. Löffler, S. Höfling, L. Worschech, and A. Forchel, J. Appl. Phys. 107, 096106 (2010).

[18] M. Syperek, A. Musiał, G. Sęk, P. Podemski, J. Misiewicz, A. Löffler, S. Höfling, L. Worschech, and A. Forchel, AIP Conf. Proc. 1399, 563 (2011).

[19] P. Poloczek, G. Sęk, J. Misiewicz, A. Löffler, J. P. Reithmaier, and A. Forchel, J. Appl. Phys. 100, 013503 (2006).

[20] A. Löffler, J. P. Reithmaier, A. Forchel, A. Sauerwald, D. Peskes, T. Kümmell, and G. Bacher, J. Crystal Growth 286, 6 (2006).

[21] M. Syperek, M. Baranowski, G. Sęk, J. Misiewicz, A. Löffler, S. Höfling, S. Reitzenstein, M. Kamp, and A. Forchel, Phys. Rev. B 87, 125305 (2013).

[22] A. V. Koudinov, I. A. Akimov, Yu. G. Kusrayev, and F. Henneberger, Phys. Rev. B 70, 241305(R) (2004).

[23] Y. Léger, L. Besombes, L. Maingault, and H. Mariette, Phys. Rev. B 76, 045331 (2007).

[24] T. Belhadj, T. Amand, A. Kunold, C.-M. Simon, T. Kuroda, M. Abbarchi, T. Mano, K. Sakoda, S. Kunz, X. Marie, and B. Urbaszek, Appl. Phys. Lett. 97, 051111 (2010).

[25] Y. Ikeuchi, T. Inoue, M. Asada, Y. Harada, T. Kita, E. Taguchi, and H. Yasuda, Appl. Phys. Exp. 4, 062001 (2011).

[26] D. Roy-Guay, P. J. Poole, and S. Raymond, Semicond. Sci. Tech. 25, 045001 (2010)

[27] S. Ohno, S. Adachi, R. Kaji, S. Muto, and H. Sasakura, Appl. Phys. Lett. 98, 161912 (2011).

[28] C. Tonin, R. Hostein, V. Voliotis, R. Grousson, A. Lemaitre, and A. Martinez, Phys. Rev. B 85, 155303 (2012).

[29] P. Kaczmarkiewicz, A. Musiał, G. Sęk, P. Podemski, P. Machnikowski, and J. Misiewicz, Acta Phys. Pol. A 119, 633 (2011).

[30] T. Mano, R. Nötzel, G. J. Hamhuis, T. J. Eijkemans, and J. H. Wolter, J. Appl. Phys. 95, 109 (2004). 
[31] T. Mano, R. Nötzel, G. J. Hamhuis, T. J. Eijkemans, and J. H. Wolter, Appl. Phys. Lett. 81, 1705 (2002).

[32] A. D. Güçlü, C. J. Umrigar, Hong Jiang, and Harold U. Baranger, Phys. Rev. B 80, 201302(R) (2009).

[33] T. Guillet, R. Grousson, V. Voliotis, X. L. Wang, and M. Ogura, Phys. Rev. B 68, 045319 (2003).

[34] A. Musiał, P. Kaczmarkiewicz, G. Sęk, P. Podemski, P. Machnikowski, J. Misiewicz, S. Hein, S. Höfling, and A. Forchel, Phys. Rev. B 85, 035314 (2012).

[35] A. Musiał, G. Sęk, P. Podemski, J. Misiewicz, A. Löffler, S. Höfling, S. Reitzenstein, J. P. Reithmaier, and A. Forchel, Acta Phys. Pol. A 120, 883 (2011).

[36] J. Andrzejewski, G. Sek, E. O'Reilly, A. Fiore, and J. Misiewicz, J. Appl. Phys. 107, 073509 (2010).

[37] J. Andrzejewski, J. Comput. Phys. 249, 22 (2013).

[38] G. Sęk, P. Poloczek, K. Ryczko, J. Misiewicz, A. Löffler, J. P. Reithmaier, and A. Forchel, J. Appl. Phys. 100, 103529 (2006).

[39] G. Bester, A. Zunger, X. Wu, and D. Vanderbilt, Phys. Rev. B 74, 081305(R) (2006).

[40] A. Beya-Wakata, P.-Y. Prodhomme, and G. Bester, Phys. Rev. B 84, 195207 (2011).

[41] A. Schliwa, M. Winkelnkemper, and D. Bimberg, Phys. Rev. B 76, 205324 (2007).

[42] J. P. Reithmaier, G. Eisenstein, and A. Forchel, Proc. IEEE 95, 1779 (2007).

[43] W. Rudno-Rudziński, G. Sęk, K. Ryczko, R. Kudrawiec, J. Misiewicz, A. Somers, R. Schwertberger, J. P. Reithmaier, and A. Forchel, Appl. Phys. Lett. 86, 101904 (2005).

[44] W. Rudno-Rudziński, R. Kudrawiec, P. Podemski, G. Sęk, J. Misiewicz, A. Somers, R. Schwertberger, J. P. Reithmaier, and A. Forchel, Appl. Phys. Lett. 89, 031908 (2006).

[45] G. Sęk, P. Podemski, A. Musiał, J. Misiewicz, S. Hein, S. Höfling, and A. Forchel, J. Appl. Phys. 105, 086104 (2009).

[46] A. Musiał, P. Podemski, G. Sęk, P. Kaczmarkiewicz, J. Andrzejewski, P. Machnikowski, J. Misiewicz, S. Hein, A. Somers, S. Höfling, J. P. Reithmaier, and A. Forchel, Semicond. Sci. Tech. 27, 105022 (2012).

[47] A. Musiał, Ph.D. dissertation, Institute of Physics, Wroclaw University of Technology, Wroclaw, Poland, 2013.

[48] T. Heindel, Ch. A. Kessler, M. Rau, Ch. Schneider, M. Fürst, F. Hargart, W.-M. Schulz, M. Eichfelder, R. Roßbach, S. Nauerth, M. Lermer, H. Weier, M. Jetter, M. Kamp, S. Reitzenstein, S. Höfling, P. Michler, H. Weinfurter, and A. Forchel, New J. Phys. 14, 083001 (2012).

[49] M. Bayer, G. Ortner, O. Stern, A. Kuther, A. A. Gorbunov, A. Forchel, P. Hawrylak, S. Fafard, K. Hinzer, T. L. Reinecke, S. N. Walck, J. P. Reithmaier, F. Klopf, and F. Schäfer, Phys. Rev. B 65, 195315 (2002).

[50] E. Kadantsev and P. Hawrylak, Phys. Rev. B 81, 045311 (2010).

[51] G. Bester, S. Nair, and A. Zunger, Phys. Rev. B 67, 161306(R) (2003).

[52] A. Högele, S. Seidl, M. Kroner, K. Karrai, R. J. Warburton, B. D. Gerardot, and P. M. Petroff, Phys. Rev. Lett. 93, 217401 (2004).

[53] A. I. Tartakovskii, M. N. Makhonin, I. R. Sellers, J. Cahill, A. D. Andreev, D. M. Whittaker, J.-P. R. Wells, A. M. Fox, D. J. Mowbray, M. S. Skolnick, K. M. Groom, M. J. Steer, H. Y. Liu, and M. Hopkinson, Phys. Rev. B 70, 193303 (2004).

[54] W. Langbein, P. Borri, U. Woggon, V. Stavarache, D. Reuter, and A. D. Wieck, Phys. Rev. B 69, 161301(R) (2004).
[55] Y. H. Huo, A. Rastelli, and O. G. Schmidt, Appl. Phys. Lett. 102, 152105 (2013).

[56] E. Stock, T. Warming, I. Ostapenko, S. Rodt, A. Schliwa, J. A. Töfflinger, A. Lochmann, A. I. Toropov, S. A. Moshchenko, D. V. Dmitriev, V. A. Haisler, and D. Bimberg, Appl. Phys. Lett. 96, 093112 (2010).

[57] T. Mano, M. Abbarchi, T. Kuroda, B. McSkimming, A. Ohtake, K. Mitsuishi, and K. Sakoda, Appl. Phys. Exp. 3, 065203 (2010).

[58] J. Treu, C. Schneider, A. Huggenberger, T. Braun, S. Reitzenstein, S. Höfling, and M. Kamp, Appl. Phys. Lett. 101, 022102 (2012).

[59] N. Akopian, N. H. Lindner, E. Poem, Y. Berlatzky, J. Avron, D. Gershoni, B. D. Gerardot, and P. M. Petroff, Phys. Rev. Lett. 96, 130501 (2006)

[60] O. Benson, Ch. Santori, M. Pelton, and Y. Yamamoto, Phys. Rev. Lett. 84, 2513 (2000).

[61] C. H. Bennett and G. Brassard, Proceedings of IEEE International Conference on Computers, Systems and Signal Processing, Bangalore, India, December 10-12, 1984, p. 175 , http://www.cs.ucsb.edu/ chong/290N-W06/BB84.pdf.

[62] E. Waks, K. Inoue, Ch. Santori, D. Fattal, J. Vuckovic, G. S. Solomon, and Y. Yamamoto, Nature 420, 762 (2002).

[63] H.-J. Briegel, W. Dür, J. I. Cirac, and P. Zoller, Phys. Rev. Lett. 81, 5932 (1998).

[64] E. Knill, R. Laflamme, and G. J. Milburn, Nature 409, 46 (2001),

[65] The estimated error bars for the largest observed FSS values are related to the fact that respective emission lines were not well isolated and possibly influenced by the QD charge environment, i.e., the line with FSS $=16 \mu \mathrm{eV}$ shows random changes in emission energy possibly due to spectral diffusion effects and the line with FSS $=14 \mu \mathrm{eV}$ is accompanied by a low-energy sideband. Their spectral proximity is only coincidental without any physical meaning. Their spectral position is close to the maximum of the QD ensemble emission energy distribution, which makes such coincidence more likely in this spectral range.

[66] R. Seguin, A. Schliwa, S. Rodt, K. Pötschke, U. W. Pohl, and D. Bimberg, Phys. Rev. Lett. 95, 257402 (2005).

[67] L. He, M. Gong, Ch.-F. Li, G.-C. Guo, and A. Zunger, Phys. Rev. Lett. 101, 157405 (2008).

[68] N. Chauvin, B. Salem, G. Bremond, G. Guillot, C. Bru-Chevallier, and M. Gendry, J. Appl. Phys. 100, 073702 (2006).

[69] O. Stier, M. Grundmann, and D. Bimberg, Phys. Rev. B 59, 5688 (1999).

[70] M. Bayer, S. N. Walck, T. L. Reinecke, and A. Forchel, Phys. Rev. B 57, 6584 (1998).

[71] S. N. Walck and T. L. Reinecke, Phys. Rev. B 57, 9088 (1998).

[72] M. Bayer, A. Kuther, A. Forchel, A. Gorbunov, V. B. Timofeev, F. Schäfer, J. P. Reithmaier, T. L. Reinecke, and S. N. Walck, Phys. Rev. Lett. 82, 1748 (1999).

[73] T. Nakaoka, T. Saito, J. Tatebayashi, S. Hirose, T. Usuki, N. Yokoyama, and Y. Arakawa, Phys. Rev. B 71, 205301 (2005).

[74] Y. Toda, S. Shinomori, K. Suzuki, and Y. Arakawa, Appl. Phys. Lett. 73, 517 (1998).

[75] W. Sheng, S. J. Xu, and P. Hawrylak, Phys. Rev. B 77, 241307(R) (2008).

[76] W. Sheng, Appl. Phys. Lett. 96, 133102 (2010).

[77] G. Medeiros-Ribeiro, M. V. B. Pinheiro, V. L. Pimentel, and E. Marega, Appl. Phys. Lett. 80, 4229 (2002). 